Pacific

Journal of

Mathematics

\title{
A QUOTIENT OF THE BRAID GROUP RELATED TO PSEUDOSYMMETRIC BRAIDED CATEGORIES
}

Florin Panaite AND Mihai D. StaiC 


\title{
A QUOTIENT OF THE BRAID GROUP RELATED TO PSEUDOSYMMETRIC BRAIDED CATEGORIES
}

\author{
Florin Panaite And Mihai D. Staic
}

\begin{abstract}
Motivated by the recent concept of a pseudosymmetric braided monoidal category, we define the pseudosymmetric group $\mathrm{PS}_{\boldsymbol{n}}$ to be the quotient of the braid group $B_{n}$ by the relations $\sigma_{i} \sigma_{i+1}^{-1} \sigma_{i}=\sigma_{i+1} \sigma_{i}^{-1} \sigma_{i+1}$ with $1 \leq i \leq n-2$. It turns out that $\mathbf{P S}_{n}$ is isomorphic to the quotient of $B_{n}$ by the commutator subgroup $\left[P_{n}, P_{n}\right]$ of the pure braid group $P_{n}$ (which amounts to saying that $\left[P_{n}, P_{n}\right]$ coincides with the normal subgroup of $B_{n}$ generated by the elements $\left[\sigma_{i}^{2}, \sigma_{i+1}^{2}\right]$ with $1 \leq i \leq n-2$ ), and that $\mathrm{PS}_{n}$ is a linear group.
\end{abstract}

\section{Introduction}

A symmetric category consists of a monoidal category $\mathscr{b}$ equipped with a family of natural isomorphisms $c_{X, Y}: X \otimes Y \rightarrow Y \otimes X$ satisfying natural "bilinearity" conditions together with the symmetry relation $c_{Y, X} \circ c_{X, Y}=\operatorname{id}_{X \otimes Y}$ for all $X, Y \in \mathscr{C}$. This concept was generalized by Joyal and Street [1993] by dropping this symmetry relation from the axioms and arriving thus at the concept of braided category, of central importance in quantum group theory; see [Kassel 1995; Majid 1995].

Inspired by recently introduced categorical concepts of pure-braided structures [Staic 2004] and twines [Bruguières 2006], Panaite, Staic and Van Oystaeyen [Panaite et al. 2009] defined the concept of pseudosymmetric braiding to generalize symmetric braidings. A braiding $c$ on a strict monoidal category $\mathscr{C}$ is pseudosymmetric if it satisfies the modified braid relation

$\left(c_{Y, Z} \otimes \mathrm{id}_{X}\right) \circ\left(\mathrm{id}_{Y} \otimes c_{Z, X}^{-1}\right) \circ\left(c_{X, Y} \otimes \mathrm{id}_{Z}\right)=\left(\mathrm{id}_{Z} \otimes c_{X, Y}\right) \circ\left(c_{Z, X}^{-1} \otimes \mathrm{id}_{Y}\right) \circ\left(\operatorname{id}_{X} \otimes c_{Y, Z}\right)$

for all $X, Y, Z \in \mathscr{C}$. The main result in [Panaite et al. 2009] asserts that, if $H$ is a Hopf algebra with bijective antipode, then the canonical braiding of the YetterDrinfeld category ${ }_{H} \mathrm{y}^{H}{ }^{H}$ is pseudosymmetric if and only if $H$ is commutative and cocommutative.

MSC2000: primary 20F36; secondary 18D10.

Keywords: braid group, symmetric group, braided categories, pseudosymmetric braidings.

Research partially supported by the CNCSIS project "Hopf algebras, cyclic homology and monoidal categories", contract number 560/2009. 
It is well known that, at several levels, braided categories correspond to the braid groups $B_{n}$, while symmetric categories correspond to the symmetric groups $S_{n}$. It is natural to expect that there exist some groups corresponding, in the same way, to pseudosymmetric braided categories. Indeed, it is clear that these groups, denoted by $\mathrm{PS}_{n}$ and called (naturally) the pseudosymmetric groups, should be the quotients of the braid groups $B_{n}$ by the relations $\sigma_{i} \sigma_{i+1}^{-1} \sigma_{i}=\sigma_{i+1} \sigma_{i}^{-1} \sigma_{i+1}$. Our aim is to study and find more explicitly the structure of these groups. We prove first that the kernel of the canonical group morphism $\mathrm{PS}_{n} \rightarrow S_{n}$ is abelian, and consequently $\mathrm{PS}_{n}$ is isomorphic to the quotient of $B_{n}$ by the commutator subgroup $\left[P_{n}, P_{n}\right]$ of the pure braid group $P_{n}$. (This amounts to saying that $\left[P_{n}, P_{n}\right]$ coincides with the normal subgroup of $B_{n}$ generated by the elements $\left[\sigma_{i}^{2}, \sigma_{i+1}^{2}\right]$ with $1 \leq i \leq n-2$.)

There exist similarities, but also differences, between braid groups and pseudosymmetric groups. Bigelow [2001] and Krammer [2002] proved that braid groups are linear, and we show that so are pseudosymmetric groups. More precisely, we prove that the Lawrence-Krammer representation of $B_{n}$ induces a representation of $\mathrm{PS}_{n}$ if the parameter $q$ is chosen to be 1 , and that this representation of $\mathrm{PS}_{n}$ is faithful over $\mathbb{R}\left[t^{ \pm 1}\right]$. On the other hand, although $\mathrm{PS}_{n}$ is an infinite group, like $B_{n}$, it does have nontrivial elements of finite order, unlike $B_{n}$.

\section{Preliminaries}

Definition 1.1 [Panaite et al. 2007]. Let $\mathscr{C}$ be a strict monoidal category and let $T_{X, Y}: X \otimes Y \rightarrow X \otimes Y$ be a family of natural isomorphisms in $\mathscr{C}$. We call $T$ a strong twine if, for all $X, Y, Z \in \mathscr{C}$,

$$
\begin{aligned}
& T_{I, I}=\mathrm{id}_{I}, \quad\left(T_{X, Y} \otimes \mathrm{id}_{Z}\right) \circ T_{X \otimes Y, Z}=\left(\operatorname{id}_{X} \otimes T_{Y, Z}\right) \circ T_{X, Y \otimes Z}, \\
& \left(T_{X, Y} \otimes \mathrm{id}_{Z}\right) \circ\left(\mathrm{id}_{X} \otimes T_{Y, Z}\right)=\left(\operatorname{id}_{X} \otimes T_{Y, Z}\right) \circ\left(T_{X, Y} \otimes \mathrm{id}_{Z}\right) .
\end{aligned}
$$

Definition 1.2 [Panaite et al. 2009]. Let $\mathscr{C}$ be a strict monoidal category and $c$ a braiding on $\mathscr{C}$. We say that $c$ is pseudosymmetric if, for all $X, Y, Z \in \mathscr{C}$,

$$
\begin{aligned}
\left(c_{Y, Z} \otimes \mathrm{id}_{X}\right) \circ\left(\operatorname{id}_{Y} \otimes c_{Z, X}^{-1}\right) & \left(c_{X, Y} \otimes \mathrm{id}_{Z}\right) \\
& =\left(\operatorname{id}_{Z} \otimes c_{X, Y}\right) \circ\left(c_{Z, X}^{-1} \otimes \operatorname{id}_{Y}\right) \circ\left(\operatorname{id}_{X} \otimes c_{Y, Z}\right) .
\end{aligned}
$$

In this case we say that $\mathscr{C}$ is a pseudosymmetric braided category.

The next proposition, a key result in [Panaite et al. 2009], led to the introduction of the concept of pseudosymmetric braiding. Here, it will serve as a source of inspiration for a certain key result for braids, Proposition 2.1.

Proposition 1.3 [Panaite et al. 2009]. Let $\mathscr{C}$ be a strict monoidal category and $c$ a braiding on $\mathscr{C}$. Then the double braiding $T_{X, Y}:=c_{Y, X} \circ c_{X, Y}$ is a strong twine if and only if $c$ is pseudosymmetric. 


\section{Defining relations for $\mathbf{P S}_{n}$}

Let $n \geq 3$ be a natural number. We denote by $B_{n}$ the braid group on $n$ strands, with its usual presentation by generators $\sigma_{i}$ with $1 \leq i \leq n-1$ and relations

$$
\begin{array}{ccrl}
\sigma_{i} \sigma_{j} & =\sigma_{j} \sigma_{i} & & \text { if }|i-j| \geq 2, \\
\sigma_{i} \sigma_{i+1} \sigma_{i} & =\sigma_{i+1} \sigma_{i} \sigma_{i+1} & & \text { if } 1 \leq i \leq n-2 .
\end{array}
$$

We begin with the analogue for braids of Proposition 1.3:

Proposition 2.1. For all $1 \leq i \leq n-2$, the relations

$$
\begin{aligned}
\sigma_{i} \sigma_{i+1}^{-1} \sigma_{i} & =\sigma_{i+1} \sigma_{i}^{-1} \sigma_{i+1}, \\
\sigma_{i}^{2} \sigma_{i+1}^{2} & =\sigma_{i+1}^{2} \sigma_{i}^{2}
\end{aligned}
$$

are equivalent in $B_{n}$.

Proof. We show first that (4) implies (5):

$$
\begin{aligned}
\sigma_{i}^{2} \sigma_{i+1}^{2} & =\sigma_{i} \sigma_{i+1}^{-1} \sigma_{i+1} \sigma_{i} \sigma_{i+1} \sigma_{i+1} \\
& \stackrel{(3)}{=} \sigma_{i} \sigma_{i+1}^{-1} \sigma_{i} \sigma_{i+1} \sigma_{i} \sigma_{i+1} \stackrel{(3),(4)}{=} \sigma_{i+1} \sigma_{i}^{-1} \sigma_{i+1} \sigma_{i} \sigma_{i+1} \sigma_{i} \\
& \stackrel{(3)}{=} \sigma_{i+1} \sigma_{i}^{-1} \sigma_{i} \sigma_{i+1} \sigma_{i} \sigma_{i}=\sigma_{i+1}^{2} \sigma_{i}^{2} .
\end{aligned}
$$

Conversely, we prove that (5) implies (4):

$$
\begin{aligned}
& \sigma_{i} \sigma_{i+1}^{-1} \sigma_{i}=\sigma_{i} \sigma_{i+1}^{-2} \sigma_{i}^{-1} \sigma_{i} \sigma_{i+1} \sigma_{i} \\
& \stackrel{(3)}{=} \sigma_{i} \sigma_{i+1}^{-2} \sigma_{i}^{-1} \sigma_{i+1} \sigma_{i} \sigma_{i+1} \\
&=\sigma_{i} \sigma_{i+1}^{-2} \sigma_{i}^{-2} \sigma_{i} \sigma_{i+1} \sigma_{i} \sigma_{i+1} \stackrel{(3),(5)}{=} \sigma_{i} \sigma_{i}^{-2} \sigma_{i+1}^{-2} \sigma_{i+1} \sigma_{i} \sigma_{i+1}^{2} \\
&=\sigma_{i}^{-1} \sigma_{i+1}^{-1} \sigma_{i} \sigma_{i+1}^{2} \\
&=\sigma_{i+1} \sigma_{i+1}^{-1} \sigma_{i}^{-1} \sigma_{i+1}^{-1} \sigma_{i} \sigma_{i+1}^{2} \\
& \stackrel{(3)}{=} \sigma_{i+1} \sigma_{i}^{-1} \sigma_{i+1}^{-1} \sigma_{i}^{-1} \sigma_{i} \sigma_{i+1}^{2} \\
&=\sigma_{i+1} \sigma_{i}^{-1} \sigma_{i+1} .
\end{aligned}
$$

Definition 2.2. For a natural number $n \geq 3$, we define the pseudosymmetric group $\mathrm{PS}_{n}$ as the group with generators $\sigma_{i}$ for $1 \leq i \leq n-1$, and relations (2), (3) and (4), or equivalently (2), (3) and (5).

Proposition 2.3. For $1 \leq i \leq n-2$, consider the elements

$$
p_{i}:=\sigma_{i} \sigma_{i+1}^{-1} \text { and } q_{i}:=\sigma_{i}^{-1} \sigma_{i+1}
$$

in $\mathrm{PS}_{n}$. Then, in $\mathrm{PS}_{n}$, we have

$$
p_{i}^{3}=q_{i}^{3}=\left(p_{i} q_{i}\right)^{3}=1 \quad \text { for all } 1 \leq i \leq n-2 .
$$


Proof. The relations $p_{i}^{3}=1$ and $q_{i}^{3}=1$ follow immediately from (4); actually each of them is equivalent to (4). Now we compute

$$
\begin{aligned}
\left(p_{i} q_{i}\right)^{2} & =\left(\sigma_{i} \sigma_{i+1}^{-1} \sigma_{i}^{-1} \sigma_{i+1}\right)^{2} \\
& =\sigma_{i} \sigma_{i+1}^{-1} \sigma_{i}^{-1} \sigma_{i+1} \sigma_{i} \sigma_{i+1}^{-1} \sigma_{i}^{-1} \sigma_{i+1} \\
& =\sigma_{i} \sigma_{i+1}^{-1} \sigma_{i}^{-1} \sigma_{i+1} \sigma_{i} \sigma_{i+1} \sigma_{i+1}^{-2} \sigma_{i}^{-1} \sigma_{i+1} \\
& \stackrel{(3)}{=} \sigma_{i}^{2} \sigma_{i+1}^{-2} \sigma_{i}^{-1} \sigma_{i+1} \\
& \stackrel{(5)}{=} \sigma_{i+1}^{-2} \sigma_{i} \sigma_{i+1} \\
& =\sigma_{i+1}^{-2} \sigma_{i} \sigma_{i+1} \sigma_{i} \sigma_{i}^{-1} \\
& \stackrel{(3)}{=} \sigma_{i+1}^{-1} \sigma_{i} \sigma_{i+1} \sigma_{i}^{-1}=\left(p_{i} q_{i}\right)^{-1},
\end{aligned}
$$

and so $\left(p_{i} q_{i}\right)^{3}=1$.

Consider now the symmetric group $S_{n}$ with its usual presentation by generators $s_{i}$ with $1 \leq i \leq n-1$ and relations (2), (3) and $s_{i}^{2}=1$ for all $1 \leq i \leq n-1$. We denote by $\pi: B_{n} \rightarrow S_{n}, \beta: B_{n} \rightarrow \mathrm{PS}_{n}$ and $\alpha: \mathrm{PS}_{n} \rightarrow S_{n}$ the canonical surjective group homomorphisms given by $\pi\left(\sigma_{i}\right)=s_{i}, \alpha\left(\sigma_{i}\right)=s_{i}$ and $\beta\left(\sigma_{i}\right)=\sigma_{i}$ for all $1 \leq i \leq n-1$. Obviously we have $\pi=\alpha \circ \beta$; hence in particular we obtain $\operatorname{Ker}(\alpha)=\beta(\operatorname{Ker}(\pi))$. We denote as usual $\operatorname{Ker}(\pi)=P_{n}$, the pure braid group on $n$ strands. It is well known (see [Kassel and Turaev 2008, page 21]) that $P_{n}$ is generated by the elements

$$
a_{i j}:=\sigma_{j-1} \sigma_{j-2} \cdots \sigma_{i+1} \sigma_{i}^{2} \sigma_{i+1}^{-1} \cdots \sigma_{j-2}^{-1} \sigma_{j-1}^{-1} \quad \text { for } 1 \leq i<j \leq n
$$

that satisfy certain relations, of which we will use only one, namely, that for $1 \leq i<j \leq n$ and $1 \leq r<s \leq n$,

$$
a_{i j} a_{r s}=a_{r s} a_{i j} \quad \text { if } s<i \text { or } i<r<s<j .
$$

Alternatively, $P_{n}$ is generated by the elements

$$
b_{i j}:=\sigma_{j-1}^{-1} \sigma_{j-2}^{-1} \cdots \sigma_{i+1}^{-1} \sigma_{i}^{2} \sigma_{i+1} \cdots \sigma_{j-2} \sigma_{j-1} \quad \text { for } 1 \leq i<j \leq n .
$$

It is easy to see that in $B_{n}$ we have

$$
\sigma_{i+1} \sigma_{i}^{2} \sigma_{i+1}^{-1}=\sigma_{i}^{-1} \sigma_{i+1}^{2} \sigma_{i} \quad \text { and } \quad \sigma_{i+1}^{-1} \sigma_{i}^{2} \sigma_{i+1}=\sigma_{i} \sigma_{i+1}^{2} \sigma_{i}^{-1},
$$

and by using repeatedly these relations we obtain an equivalent description of the elements $a_{i j}$ and $b_{i j}$ :

$$
\begin{array}{ll}
a_{i j}=\sigma_{i}^{-1} \sigma_{i+1}^{-1} \cdots \sigma_{j-2}^{-1} \sigma_{j-1}^{2} \sigma_{j-2} \cdots \sigma_{i+1} \sigma_{i} & \text { for } 1 \leq i<j \leq n, \\
b_{i j}=\sigma_{i} \sigma_{i+1} \cdots \sigma_{j-2} \sigma_{j-1}^{2} \sigma_{j-2}^{-1} \cdots \sigma_{i+1}^{-1} \sigma_{i}^{-1} & \text { for } 1 \leq i<j \leq n .
\end{array}
$$


Now, for all $1 \leq i<j \leq n$, we define $A_{i, j}$ and $B_{i, j}$ as the elements in $\mathrm{PS}_{n}$ given by $A_{i, j}:=\beta\left(a_{i j}\right)$ and $B_{i, j}:=\beta\left(b_{i j}\right)$. From the discussion above it follows that $\operatorname{Ker}(\alpha)$ is generated by $\left\{A_{i, j}\right\}_{1 \leq i<j \leq n}$ and also by $\left\{B_{i, j}\right\}_{1 \leq i<j \leq n}$.

Lemma 2.4. The following relations hold in $\mathrm{PS}_{n}$ for $1 \leq i<j<n$ :

$$
\begin{aligned}
& A_{i, j+1}=\sigma_{j} A_{i, j} \sigma_{j}^{-1}, \\
& B_{i, j+1}=\sigma_{j}^{-1} B_{i, j} \sigma_{j} .
\end{aligned}
$$

Proof. These relations are consequences of corresponding relations in $B_{n}$ for the $a_{i j}$ and $b_{i j}$, which in turn follow immediately from (8) and (10).

Lemma 2.5. For all $i, j \in\{1,2, \ldots, n\}$ with $i+1<j$, we have in $\mathrm{PS}_{n}$

$$
\begin{aligned}
& A_{i, j}=\sigma_{i} A_{i+1, j} \sigma_{i}^{-1}, \\
& B_{i, j}=\sigma_{i}^{-1} B_{i+1, j} \sigma_{i} .
\end{aligned}
$$

Proof. We prove (16), while (17) is similar and left to the reader. Note that in $\mathrm{PS}_{n}$ we have $\sigma_{i+1}^{-1} \sigma_{i}^{2} \sigma_{i+1}=\sigma_{i+1} \sigma_{i}^{2} \sigma_{i+1}^{-1}$, which together with the second of (11) implies $\sigma_{i} \sigma_{i+1}^{2} \sigma_{i}^{-1}=\sigma_{i+1} \sigma_{i}^{2} \sigma_{i+1}^{-1}$; hence

$$
\begin{aligned}
A_{i, j} & =\sigma_{j-1} \sigma_{j-2} \cdots\left(\sigma_{i+1} \sigma_{i}^{2} \sigma_{i+1}^{-1}\right) \cdots \sigma_{j-2}^{-1} \sigma_{j-1}^{-1} \\
& =\sigma_{j-1} \sigma_{j-2} \cdots\left(\sigma_{i} \sigma_{i+1}^{2} \sigma_{i}^{-1}\right) \cdots \sigma_{j-2}^{-1} \sigma_{j-1}^{-1} \\
& =\sigma_{i} \sigma_{j-1} \sigma_{j-2} \cdots \sigma_{i+1}^{2} \cdots \sigma_{j-2}^{-1} \sigma_{j-1}^{-1} \sigma_{i}^{-1}=\sigma_{i} A_{i+1, j} \sigma_{i}^{-1} .
\end{aligned}
$$

Proposition 2.6. For all $1 \leq i<j \leq n$, we have $A_{i, j}=B_{i, j}$ in $\mathrm{PS}_{n}$.

Proof. We use (16) repeatedly:

$$
\begin{aligned}
A_{i, j}=\sigma_{i} A_{i+1, j} \sigma_{i}^{-1} & =\sigma_{i} \sigma_{i+1} A_{i+2, j} \sigma_{i+1}^{-1} \sigma_{i}^{-1} \\
& \cdots \\
& =\sigma_{i} \sigma_{i+1} \cdots \sigma_{j-2} A_{j-1, j} \sigma_{j-2}^{-1} \cdots \sigma_{i+1}^{-1} \sigma_{i}^{-1} \\
& =\sigma_{i} \sigma_{i+1} \cdots \sigma_{j-2} \sigma_{j-1}^{2} \sigma_{j-2}^{-1} \cdots \sigma_{i+1}^{-1} \sigma_{i}^{-1} \stackrel{(13)}{=} B_{i, j} .
\end{aligned}
$$

Lemma 2.7. For all $1 \leq i<j \leq n$ and $1 \leq h \leq k<n$, we have in $\mathrm{PS}_{n}$

$$
\begin{aligned}
A_{i, j} \sigma_{i}^{2} & =\sigma_{i}^{2} A_{i, j}, \\
A_{h, k+1} \sigma_{k}^{2} & =\sigma_{k}^{2} A_{h, k+1} .
\end{aligned}
$$

Proof. Note first that (18) is obvious for $j=i+1$. Assume that $i+1<j$; using the fact that $A_{r, s}=B_{r, s}$ for all $r, s$, we compute

$$
A_{i, j} \sigma_{i}^{2} \stackrel{(16)}{=} \sigma_{i} A_{i+1, j} \sigma_{i}=\sigma_{i} B_{i+1, j} \sigma_{i} \stackrel{(17)}{=} \sigma_{i}^{2} B_{i, j}=\sigma_{i}^{2} A_{i, j} .
$$


Note also that (19) is obvious for $h=k$. Assume that $h<k$; using again $A_{r, s}=B_{r, s}$ for all $r, s$, we compute

$$
A_{h, k+1} \sigma_{k}^{2} \stackrel{(14)}{=} \sigma_{k} A_{h, k} \sigma_{k}=\sigma_{k} B_{h, k} \sigma_{k} \stackrel{(15)}{=} \sigma_{k}^{2} B_{h, k+1}=\sigma_{k}^{2} A_{h, k+1} .
$$

\section{The structure of $\mathbf{P S}_{n}$}

We denote by $\mathfrak{P}_{n}$ the kernel of the morphism $\alpha: \mathrm{PS}_{n} \rightarrow S_{n}$ defined above.

Proposition 3.1. $\mathfrak{P}_{n}$ is an abelian group.

Proof. It is enough to prove that any two elements $A_{i, j}$ and $A_{k, l}$ commute in $\mathrm{PS}_{n}$. We only have to analyze the following seven cases for the numbers $i, j, k, l$ :

(i) $i<j<k<l$. This is an obvious consequence of (9).

(ii) $i<j=k<l$. We write

$$
\begin{aligned}
& A_{i, j}=\sigma_{i}^{-1} \sigma_{i+1}^{-1} \cdots \sigma_{j-2}^{-1} \sigma_{j-1}^{2} \sigma_{j-2} \cdots \sigma_{i+1} \sigma_{i}, \\
& A_{j, l}=\sigma_{l-1} \sigma_{l-2} \cdots \sigma_{j+1} \sigma_{j}^{2} \sigma_{j+1}^{-1} \cdots \sigma_{l-2}^{-1} \sigma_{l-1}^{-1},
\end{aligned}
$$

and we obtain $A_{i, j} A_{j, l}=A_{j, l} A_{i, j}$ by using (2) and the fact that $\sigma_{j-1}^{2}$ and $\sigma_{j}^{2}$ commute in $\mathrm{PS}_{n}$.

(iii) $i<k<j<l$. This follows since $A_{k, l}=B_{k, l}$ in $\mathrm{PS}_{n}$ (Proposition 2.6), and $a_{i j}$ and $b_{k l}$ commute in $P_{n}$ if $i<k<j<l$, which is easily seen geometrically.

(iv) $i=k<j=l$. This is trivial.

(v) $i<k<l<j$. This is an obvious consequence of (9).

(vi) $i=k<j<l$. In case $j=i+1$, we have $A_{i, j}=\sigma_{i}^{2}$ and so we obtain $A_{i, j} A_{i, l}=A_{i, l} A_{i, j}$ by using (18); assuming now $i+1<j$, by using repeatedly (16) we can compute

$$
\begin{aligned}
A_{i, j} A_{i, l} & =\sigma_{i} A_{i+1, j} A_{i+1, l} \sigma_{i}^{-1} \\
& =\sigma_{i} \sigma_{i+1} A_{i+2, j} A_{i+2, l} \sigma_{i+1}^{-1} \sigma_{i}^{-1} \\
& \cdots \\
& =\sigma_{i} \sigma_{i+1} \cdots \sigma_{j-2} A_{j-1, j} A_{j-1, l} \sigma_{j-2}^{-1} \cdots \sigma_{i+1}^{-1} \sigma_{i}^{-1},
\end{aligned}
$$

and similarly

$$
A_{i, l} A_{i, j}=\sigma_{i} \sigma_{i+1} \cdots \sigma_{j-2} A_{j-1, l} A_{j-1, j} \sigma_{j-2}^{-1} \cdots \sigma_{i+1}^{-1} \sigma_{i}^{-1}
$$

these are equal since $A_{j-1, j}=\sigma_{j-1}^{2}$ and by (18), $\sigma_{j-1}^{2} A_{j-1, l}=A_{j-1, l} \sigma_{j-1}^{2}$. 
(vii) $i<k<j=l$. In case $j=k+1$, we have $A_{k, j}=\sigma_{k}^{2}$ and so we obtain $A_{i, j} A_{k, j}=A_{k, j} A_{i, j}$ by using (19); assuming now $k+1<j$, by repeatedly using (14) we can compute

$$
\begin{aligned}
A_{i, j} A_{k, j} & =\sigma_{j-1} A_{i, j-1} A_{k, j-1} \sigma_{j-1}^{-1} \\
& =\sigma_{j-1} \sigma_{j-2} A_{i, j-2} A_{k, j-2} \sigma_{j-2}^{-1} \sigma_{j-1}^{-1} \\
& \cdots \\
& =\sigma_{j-1} \sigma_{j-2} \cdots \sigma_{k+1} A_{i, k+1} A_{k, k+1} \sigma_{k+1}^{-1} \cdots \sigma_{j-2}^{-1} \sigma_{j-1}^{-1},
\end{aligned}
$$

and similarly

$$
A_{k, j} A_{i, j}=\sigma_{j-1} \sigma_{j-2} \cdots \sigma_{k+1} A_{k, k+1} A_{i, k+1} \sigma_{k+1}^{-1} \cdots \sigma_{j-2}^{-1} \sigma_{j-1}^{-1} ;
$$

these are equal since $A_{k, k+1}=\sigma_{k}^{2}$ and by (19), $A_{i, k+1} \sigma_{k}^{2}=\sigma_{k}^{2} A_{i, k+1}$.

Let $G$ be a group. If $x, y \in G$ we denote by $[x, y]:=x^{-1} y^{-1} x y$ the commutator of $x$ and $y$, and by $G^{\prime}$ the commutator subgroup of $G$ (the subgroup of $G$ generated by all commutators $[x, y]$ ), which is the smallest normal subgroup $N$ of $G$ with the property that $G / N$ is abelian. Moreover, $G^{\prime}$ is a characteristic subgroup of $G$, that is, $\theta\left(G^{\prime}\right)=G^{\prime}$ for all $\theta \in \operatorname{Aut}(G)$.

Proposition 3.2. $\mathfrak{P}_{n} \simeq P_{n} / P_{n}^{\prime} \simeq \mathbb{Z}^{n(n-1) / 2}$.

Proof. For $1 \leq i \leq n-2$ we define $t_{i} \in P_{n}$ by $t_{i}:=\left[\sigma_{i}^{2}, \sigma_{i+1}^{2}\right]=\left[a_{i, i+1}, a_{i+1, i+2}\right]$. These elements are the relators added to the ones of $B_{n}$ in order to obtain $\mathrm{PS}_{n}$; therefore, as a particular case of a general fact about groups given by generators and relations (see for instance [Coxeter and Moser 1972, page 2]), the kernel of the map $\beta: B_{n} \rightarrow \mathrm{PS}_{n}$ defined above coincides with the normal subgroup of $B_{n}$ generated by $\left\{t_{i}\right\}_{1 \leq i \leq n-2}$, which will be denoted by $L_{n}$. We obviously have $L_{n} \subseteq P_{n}$, and if we consider the map $\beta$ restricted to $P_{n}$, we have a surjective morphism $P_{n} \rightarrow \mathfrak{P}_{n}$ with kernel $L_{n}$, so $\mathfrak{P}_{n} \simeq P_{n} / L_{n}$. By Proposition 3.1 we know that $\mathfrak{P}_{n}$ is abelian, so we obtain $P_{n}^{\prime} \subseteq L_{n}$. On the other hand, since $P_{n}^{\prime}$ is characteristic in $P_{n}$ and $P_{n}$ is normal in $B_{n}$, it follows (see [Suzuki 1982, Proposition 6.14]) that $P_{n}^{\prime}$ is normal in $B_{n}$, and since $t_{1}, \ldots, t_{n-2} \in P_{n}^{\prime}$ and $L_{n}$ is the normal subgroup of $B_{n}$ generated by $\left\{t_{i}\right\}_{1 \leq i \leq n-2}$, we obtain $L_{n} \subseteq P_{n}^{\prime}$. Thus, we have obtained $L_{n}=P_{n}^{\prime}$ and so $\mathfrak{P}_{n} \simeq P_{n} / P_{n}^{\prime}$. On the other hand, it is well known that $P_{n} / P_{n}^{\prime} \simeq \mathbb{Z}^{n(n-1) / 2}$; see for instance [Kassel and Turaev 2008, Corollary 1.20].

As a consequence of the equality $L_{n}=P_{n}^{\prime}$, we obtain $B_{n} / P_{n}^{\prime}$ :

Corollary 3.3. $\mathrm{PS}_{n} \simeq B_{n} / P_{n}^{\prime}$.

The extension with abelian kernel $1 \rightarrow \mathfrak{P}_{n} \rightarrow \mathrm{PS}_{n} \rightarrow S_{n} \rightarrow 1$ induces an action of $S_{n}$ on $\mathfrak{P}_{n}$, given by $\sigma \cdot a=\tilde{\sigma} a \tilde{\sigma}^{-1}$ for $\sigma \in S_{n}$ and $a \in \mathfrak{P}_{n}$, where $\tilde{\sigma}$ is an element of $\mathrm{PS}_{n}$ with $\alpha(\tilde{\sigma})=\sigma$. In particular, on generators we have $s_{k} \cdot A_{i, j}=\sigma_{k} A_{i, j} \sigma_{k}^{-1}$, 
for $1 \leq k \leq n-1$ and $1 \leq i<j \leq n$. By using some of the formulas given above, one can describe explicitly this action as

$$
\begin{aligned}
s_{k} \cdot A_{i, j} & =A_{i, j} & & \text { if } k<i-1, \\
s_{i-1} \cdot A_{i, j} & =A_{i-1, j}, & & \\
s_{i} \cdot A_{i, j} & =A_{i+1, j} & & \text { if } j-i>1 \text { and } s_{i} \cdot A_{i, i+1}=A_{i, i+1}, \\
s_{k} \cdot A_{i, j} & =A_{i, j} & & \text { if } i<k<j-1, \\
s_{j-1} \cdot A_{i, j} & =A_{i, j-1} & & \text { if } j-i>1 \text { and } s_{j-1} \cdot A_{j-1, j}=A_{j-1, j}, \\
s_{j} \cdot A_{i j} & =A_{i, j+1} & & \text { for } 1 \leq i<j<n, \\
s_{k} \cdot A_{i, j} & =A_{i, j} & & \text { if } j<k .
\end{aligned}
$$

Note that the first equality in (20c) follows by using (17) together with the fact that $A_{i, j}=B_{i, j}$ (Proposition 2.6), and the first equality in (20e) follows by an easy computation using also the fact that $A_{i, j}=B_{i, j}$. Also, one can easily see that these formulas may be expressed more compactly as follows: If $\sigma \in\left\{s_{1}, \ldots, s_{n-1}\right\}$ and $1 \leq i<j \leq n$, then $\sigma \cdot A_{i, j}=A_{\sigma(i), \sigma(j)}$, where we made the convention $A_{r, t}:=A_{t, r}$ for $t<r$. Since $s_{1}, \ldots, s_{n-1}$ generate $S_{n}$, we have found the action of $S_{n}$ on $A_{i, j}$ :

Proposition 3.4. For any $\sigma \in S_{n}$ and $1 \leq i<j \leq n$, the action of $\sigma$ on $A_{i, j}$ is given by $\sigma \cdot A_{i, j}=A_{\sigma(i), \sigma(j)}$, with the convention $A_{r, t}:=A_{t, r}$ for $t<r$.

Lemma 3.5. Let $F$ be a free $\mathbb{Z}$-module of rank $m$, and let $\left\{X_{1}, \ldots, X_{m}\right\}$ be a generating system for $F$ over $\mathbb{Z}$. Then $\left\{X_{1}, \ldots, X_{m}\right\}$ is a basis of $F$ over $\mathbb{Z}$.

Proof. Assume $X_{1}, \ldots, X_{m}$ are linearly dependent over $\mathbb{Z}$ and take $\sum_{i=1}^{m} \alpha_{i} X_{i}=0$ a nontrivial linear combination over $\mathbb{Z}$. Choose a prime number $p$ such that $\left|\alpha_{i}\right|<p$ for all $1 \leq i \leq m$, and consider $\bar{F}:=F / p F$, a linear space over the field $\mathbb{Z}_{p}=\mathbb{Z} / p \mathbb{Z}$, and $\bar{X}_{i}$, the images of the elements $X_{i}$ in $\bar{F}$. These elements generate $\bar{F}$ over $\mathbb{Z}_{p}$, and since the dimension of $\bar{F}$ over $\mathbb{Z}_{p}$ is $m$, it follows that $\left\{\bar{X}_{1}, \ldots, \bar{X}_{m}\right\}$ is a basis of $\bar{F}$ over $\mathbb{Z}_{p}$. Thus, it follows that $\alpha_{i} \equiv 0(\bmod p)$ for all $1 \leq i \leq m$, which is a contradiction because we have chosen $p$ so that $\left|\alpha_{i}\right|<p$ for all $1 \leq i \leq m$.

Proposition 3.6. In $\mathrm{PS}_{n}$, there is no element of order 2 whose image in $S_{n}$ is the transposition $s_{1}=(1,2)$. Consequently, the extension $1 \rightarrow \mathfrak{P}_{n} \rightarrow \mathrm{PS}_{n} \rightarrow S_{n} \rightarrow 1$ is not split.

Proof. Take $x \in \mathrm{PS}_{n}$ such that $\alpha(x)=s_{1}$. Since $\alpha\left(\sigma_{1}\right)=s_{1}$, we obtain that $x \sigma_{1}^{-1} \in \operatorname{Ker}(\alpha)=\mathfrak{P}_{n}$. By Proposition 3.2 and Lemma 3.5, it follows that the abelian group $\mathfrak{P}_{n}$ is freely generated by $\left\{A_{i, j}\right\}_{1 \leq i<j \leq n}$, so we can write uniquely 
$x=\prod_{1 \leq i<j \leq n} A_{i, j}^{m_{i j}} \sigma_{1}$, with $m_{i j} \in \mathbb{Z}$. We compute

$$
\begin{aligned}
x^{2} & =\left(\prod_{1 \leq i<j \leq n} A_{i, j}^{m_{i j}} \sigma_{1}\right)\left(\prod_{1 \leq i<j \leq n} A_{i, j}^{m_{i j}} \sigma_{1}\right) \\
& =\left(\prod_{1 \leq i<j \leq n} A_{i, j}^{m_{i j}}\right)\left(\sigma_{1} \prod_{1 \leq i<j \leq n} A_{i, j}^{m_{i j}} \sigma_{1}^{-1}\right) \sigma_{1}^{2} \\
& =\left(\prod_{1 \leq i<j \leq n} A_{i, j}^{m_{i j}}\right)\left(\prod_{1 \leq i<j \leq n} \sigma_{1} A_{i, j}^{m_{i j}} \sigma_{1}^{-1}\right) A_{1,2} \\
& =A_{1,2}^{2 m_{12}+1}\left(\prod_{3 \leq j \leq n} A_{1, j}^{m_{1 j}+m_{2 j}} A_{2, j}^{m_{1 j}+m_{2 j}}\right)\left(\prod_{3 \leq i<j \leq n} A_{i, j}^{2 m_{i j}}\right),
\end{aligned}
$$

and this element cannot be trivial because $2 m_{12}+1$ cannot be 0 . Note that for the last equality we used the commutation relations

$$
\begin{array}{ll}
\sigma_{1} A_{1,2} \sigma_{1}^{-1}=A_{1,2}, & \\
\sigma_{1} A_{1, j} \sigma_{1}^{-1}=A_{2, j} & \text { for all } j \geq 3, \\
\sigma_{1} A_{2, j} \sigma_{1}^{-1}=A_{1, j} & \text { for all } j \geq 3, \\
\sigma_{1} A_{i, j} \sigma_{1}^{-1}=A_{i, j} & \text { for all } 3 \leq i<j,
\end{array}
$$

which can be easily proved by using some of the formulas given above.

Remark 3.7. As is well known [Brown 1982], any extension with abelian kernel corresponds to a 2-cocycle. Specifically, the extension $1 \rightarrow \mathfrak{P}_{n} \rightarrow \mathrm{PS}_{n} \rightarrow S_{n} \rightarrow 1$ corresponds to an element in $H^{2}\left(S_{n}, \mathbb{Z}^{n(n-1) / 2}\right)$. We illustrate this by computing explicitly the corresponding 2-cocycle for $n=3$. We consider the set-theoretical section $f: S_{3} \rightarrow \mathrm{PS}_{3}$ defined by $f(1)=1, f\left(s_{2}\right)=\sigma_{2}, f\left(s_{1}\right)=\sigma_{1}, f\left(s_{1} s_{2}\right)=\sigma_{1} \sigma_{2}$, $f\left(s_{2} s_{1}\right)=\sigma_{2} \sigma_{1}$ and $f\left(s_{2} s_{1} s_{2}\right)=\sigma_{2} \sigma_{1} \sigma_{2}$. The 2-cocycle afforded by this section is defined by $u: S_{3} \times S_{3} \rightarrow \mathfrak{P}_{3},(x, y) \mapsto f(x) f(y) f(x y)^{-1}$, and a direct computation gives its explicit formula as in Table 1 , where we have chosen an additive notation for the abelian group $\mathfrak{P}_{3} \simeq \mathbb{Z}^{3}$.

\begin{tabular}{l|cccccc} 
& 1 & $s_{2}$ & $s_{1}$ & $s_{1} s_{2}$ & $s_{2} s_{1}$ & $s_{2} s_{1} s_{2}$ \\
\hline 1 & 0 & 0 & 0 & 0 & 0 & 0 \\
$s_{2}$ & 0 & $A_{2,3}$ & 0 & 0 & $A_{2,3}$ & $A_{2,3}$ \\
$s_{1}$ & 0 & 0 & $A_{1,2}$ & $A_{1,2}$ & 0 & $A_{1,2}$ \\
$s_{1} s_{2}$ & 0 & $A_{1,3}$ & 0 & $A_{1,2}$ & $A_{1,2}+A_{1,3}$ & $A_{1,2}+A_{1,3}$ \\
$s_{2} s_{1}$ & 0 & 0 & $A_{1,3}$ & $A_{1,3}+A_{2,3}$ & $A_{2,3}$ & $A_{1,3}+A_{2,3}$ \\
$s_{2} s_{1} s_{2}$ & 0 & $A_{1,2}$ & $A_{2,3}$ & $A_{1,3}+A_{2,3}$ & $A_{1,2}+A_{1,3}$ & $A_{1,2}+A_{1,3}+A_{2,3}$ \\
\hline
\end{tabular}

Table 1. The 2-cocycle for $n=3$ associated to the section $f$. 


\section{4. $\mathrm{PS}_{n}$ is linear}

Bigelow [2001] and Krammer [2002] proved that the braid group $B_{n}$ is linear. More precisely, let $R$ be a commutative ring, let $q$ and $t$ be two invertible elements in $R$, and let $V$ be a free $R$-module of rank $n(n-1) / 2$ with a basis $\left\{x_{i, j}\right\}_{1 \leq i<j \leq n}$. Then the map $\rho: B_{n} \rightarrow \mathrm{GL}(V)$, defined by

$$
\begin{aligned}
\sigma_{k} x_{k, k+1} & =t q^{2} x_{k, k+1}, & & \\
\sigma_{k} x_{i, k} & =(1-q) x_{i, k}+q x_{i, k+1} & & \text { for } i<k, \\
\sigma_{k} x_{i, k+1} & =x_{i, k}+t q^{k-i+1}(q-1) x_{k, k+1} & & \text { for } i<k, \\
\sigma_{k} x_{k, j} & =t q(q-1) x_{k, k+1}+q x_{k+1, j} & & \text { for } k+1<j, \\
\sigma_{k} x_{k+1, j} & =x_{k, j}+(1-q) x_{k+1, j} & & \text { for } k+1<j, \\
\sigma_{k} x_{i, j} & =x_{i, j} & & \text { for } i<j<k \text { or } k+1<i<j, \\
\sigma_{k} x_{i, j} & =x_{i, j}+t q^{k-i}(q-1)^{2} x_{k, k+1} & & \text { for } i<k<k+1<j,
\end{aligned}
$$

and $\rho(x)(v)=x v$ for $x \in B_{n}$ and $v \in V$, gives a representation of $B_{n}$, and if also $R=\mathbb{R}\left[t^{ \pm 1}\right]$ and $q \in \mathbb{R} \subseteq R$ with $0<q<1$, then the representation is faithful; see [Krammer 2002].

We consider now the general formula for $\rho$, in which we take $q=1$ :

$$
\begin{aligned}
\sigma_{k} x_{k, k+1} & =t x_{k, k+1}, & & \\
\sigma_{k} x_{i, k} & =x_{i, k+1} & & \text { for } i<k, \\
\sigma_{k} x_{i, k+1} & =x_{i, k} & & \text { for } i<k, \\
\sigma_{k} x_{k, j} & =x_{k+1, j} & & \text { for } k+1<j, \\
\sigma_{k} x_{k+1, j} & =x_{k, j} & & \text { for } k+1<j, \\
\sigma_{k} x_{i, j} & =x_{i, j} & & \text { for } i<j<k \text { or } k+1<i<j, \\
\sigma_{k} x_{i, j} & =x_{i, j} & & \text { for } i<k<k+1<j .
\end{aligned}
$$

One can easily see that these formulas imply

$$
\sigma_{k}^{2} x_{k, k+1}=t^{2} x_{k, k+1} \quad \text { and } \quad \sigma_{k}^{2} x_{i, j}=x_{i, j} \quad \text { if }(i, j) \neq(k, k+1) .
$$

One can then check that $\rho\left(\sigma_{k}^{2}\right)$ commutes with $\rho\left(\sigma_{k+1}^{2}\right)$ for all $1 \leq k \leq n-2$, and so for $q=1$ it turns out that $\rho$ is a representation of $\mathrm{PS}_{n}$.

Theorem 4.1. This representation of $\mathrm{PS}_{n}$ is faithful if $R=\mathbb{R}\left[t^{ \pm 1}\right]$. Therefore, $\mathrm{PS}_{n}$ is linear.

Proof. We first prove that $A_{i, j} x_{i, j}=t^{2} x_{i, j}$, and $A_{i, j} x_{k, l}=x_{k, l}$ if $(i, j) \neq(k, l)$. We do it by induction over $|j-i|$. If $|j-i|=1$, the relations follow from the fact that $A_{i, i+1}=\sigma_{i}^{2}$. Assume the relations hold for $|j-i|=s-1$. We want to prove 
them for $|j-i|=s$. We recall that $A_{i, j}=\sigma_{j-1} A_{i, j-1} \sigma_{j-1}^{-1}$; see (14). We compute

$$
\begin{aligned}
A_{i, j} x_{i, j}=\sigma_{j-1} A_{i, j-1} \sigma_{j-1}^{-1} x_{i, j} & =\sigma_{j-1} A_{i, j-1} x_{i, j-1} \\
& =\sigma_{j-1} t^{2} x_{i, j-1} \quad \text { (by induction) } \\
& =t^{2} x_{i, j}
\end{aligned}
$$

On the other hand, if $(i, j) \neq(k, l)$ then $\sigma_{j-1}^{-1} x_{k, l}=x_{u, v}$ with $(i, j-1) \neq(u, v)$, and so

$$
\begin{aligned}
A_{i, j} x_{k, l}=\sigma_{j-1} A_{i, j-1} \sigma_{j-1}^{-1} x_{k, l} & =\sigma_{j-1} A_{i, j-1} x_{u, v} \\
& =\sigma_{j-1} x_{u, v} \quad \text { (by induction) } \\
& =\sigma_{j-1} \sigma_{j-1}^{-1} x_{k, l}=x_{k, l},
\end{aligned}
$$

as desired.

To show that the representation is faithful, take $b \in \mathrm{PS}_{n}$ such that $\rho(b)=\mathrm{id}_{V}$ and consider $\alpha(b)$, the image of $b$ in $S_{n}$. From the way $\rho$ is defined it follows that

$$
b x_{i, j}=t^{p} x_{\alpha(b)(i), \alpha(b)(j)} \text { for all } 1 \leq i<j \leq n,
$$

with $p \in \mathbb{Z}$, where we made the convention $x_{r, s}:=x_{s, r}$ if $1 \leq s<r \leq n$. Since $x_{i, j}$ is a basis in $V$ and we assumed $\rho(b)=\mathrm{id}_{V}$, we find that the permutation $\alpha(b) \in S_{n}$ has the property that if $1 \leq i<j \leq n$, then either $\alpha(b)(i)=i$ and $\alpha(b)(j)=j$ or $\alpha(b)(i)=j$ and $\alpha(b)(j)=i$. Since we assumed $n \geq 3$, the only such permutation is the trivial one. Thus, we have obtained that $b \in \operatorname{Ker}(\alpha)=\mathfrak{P}_{n}$ and so we can write $b=\prod_{1 \leq i<j \leq n} A_{i, j}^{m_{i, j}}$, with $m_{i, j} \in \mathbb{Z}$. By using the formulas given above for the action of $A_{i, j}$ on $x_{k, l}$ we immediately obtain $b x_{k, l}=t^{2 m_{k, l}} x_{k, l} \quad$ for all $1 \leq k<l \leq n$. Using again the assumption $\rho(b)=\mathrm{id}_{V}$, we obtain $t^{2 m_{k, l}}=1$ and hence $m_{k, l}=0$ for all $1 \leq k<l \leq n$, that is $b=1$, finishing the proof.

\section{Pseudosymmetric groups and pseudosymmetric braidings}

We recall from [Kassel 1995, XIII.2] that to braid groups one can associate the so-called braid category $\mathscr{B}$, a universal braided monoidal category. Similarly, we can construct a pseudosymmetric braided category $\mathscr{P} \mathscr{S}$ associated to pseudosymmetric groups. Namely, the objects of $\mathscr{P} \mathscr{S}$ are natural numbers $n \in \mathbb{N}$. The set of morphisms from $m$ to $n$ is empty if $m \neq n$ and is $\operatorname{PS}_{n}$ if $m=n$. The monoidal structure of $\mathscr{P} \mathscr{Y}$ is defined as the one for $\mathscr{B}$, and so is the braiding, namely

$$
\begin{aligned}
& c_{n, m}: n \otimes m \rightarrow m \otimes n, \\
& c_{0, n}=\mathrm{id}_{n}=c_{n, 0}, \\
& c_{n, m}=\left(\sigma_{m} \sigma_{m-1} \cdots \sigma_{1}\right)\left(\sigma_{m+1} \sigma_{m} \cdots \sigma_{2}\right) \cdots\left(\sigma_{m+n-1} \sigma_{m+n-2} \cdots \sigma_{n}\right) \quad \text { if } m, n>0 .
\end{aligned}
$$


We denote by $t_{m, n}=c_{n, m} \circ c_{m, n}$ the double braiding. In view of Proposition 1.3, to prove that $c$ is pseudosymmetric it is enough to check that, for all $m, n, p \in \mathbb{N}$,

$$
\left(t_{m, n} \otimes \mathrm{id}_{p}\right) \circ\left(\mathrm{id}_{m} \otimes t_{n, p}\right)=\left(\mathrm{id}_{m} \otimes t_{n, p}\right) \circ\left(t_{m, n} \otimes \mathrm{id}_{p}\right) .
$$

Note that $t_{m, n} \otimes \operatorname{id}_{p}$ and $\mathrm{id}_{m} \otimes t_{n, p}$ are elements in $\mathfrak{P}_{m+n+p}$, which is an abelian group, and the composition o between $t_{m, n} \otimes \mathrm{id}_{p}$ and $\mathrm{id}_{m} \otimes t_{n, p}$ is just the multiplication in the group $\mathfrak{P}_{m+n+p}$, so (21) is obviously true.

Let $\mathscr{C}$ be a strict braided monoidal category with braiding $c$, let $n$ be a natural number and let $V \in \mathscr{C}$. Consider the automorphisms $c_{1}, \ldots, c_{n-1}$ of $V^{\otimes n}$ defined by $c_{i}=\mathrm{id}_{V^{\otimes(i-1)}} \otimes c_{V, V} \otimes \mathrm{id}_{V^{\otimes(n-i-1)}}$. It is well known (see [Kassel 1995, XV.4]) that there exists a unique group morphism $\rho_{n}^{c}: B_{n} \rightarrow \operatorname{Aut}\left(V^{\otimes n}\right)$ such that $\rho_{n}^{c}\left(\sigma_{i}\right)=c_{i}$ for all $1 \leq i \leq n-1$. It is clear that, if $c$ is pseudosymmetric, then $\rho_{n}^{c}$ factorizes to a group morphism $\mathrm{PS}_{n} \rightarrow \operatorname{Aut}\left(V^{\otimes n}\right)$. Thus, pseudosymmetric braided categories provide representations of pseudosymmetric groups.

\section{References}

[Bigelow 2001] S. J. Bigelow, "Braid groups are linear", J. Amer. Math. Soc. 14:2 (2001), 471-486. MR 2002a:20043 Zbl 0988.20021

[Brown 1982] K. S. Brown, Cohomology of groups, Graduate Texts in Mathematics 87, Springer, New York, 1982. MR 83k:20002 Zbl 0584.20036

[Bruguières 2006] A. Bruguières, "Double braidings, twists and tangle invariants", J. Pure Appl. Algebra 204:1 (2006), 170-194. MR 2006k:57030 Zbl 1091.57007

[Coxeter and Moser 1972] H. S. M. Coxeter and W. O. J. Moser, Generators and relations for discrete groups, 3rd ed., Ergebnisse der Mathematik und ihrer Grenzgebiete 14, Springer, New York, 1972. MR 50 \#2313 Zbl 0239.20040

[Joyal and Street 1993] A. Joyal and R. Street, "Braided tensor categories", Adv. Math. 102:1 (1993), 20-78. MR 94m:18008 Zbl 0817.18007

[Kassel 1995] C. Kassel, Quantum groups, Graduate Texts in Mathematics 155, Springer, New York, 1995. MR 96e:17041 Zbl 0808.17003

[Kassel and Turaev 2008] C. Kassel and V. Turaev, Braid groups, Graduate Texts in Mathematics 247, Springer, New York, 2008. MR 2009e:20082 Zbl 05268073

[Krammer 2002] D. Krammer, "Braid groups are linear", Ann. of Math. (2) 155:1 (2002), 131-156. MR 2003c:20040 Zbl 1020.20025

[Majid 1995] S. Majid, Foundations of quantum group theory, Cambridge University Press, 1995. MR 97g:17016 Zbl 0857.17009

[Panaite et al. 2007] F. Panaite, M. D. Staic, and F. Van Oystaeyen, "On some classes of lazy cocycles and categorical structures”, J. Pure Appl. Algebra 209:3 (2007), 687-701. MR 2007k:16074 Zbl 1155.16030

[Panaite et al. 2009] F. Panaite, M. D. Staic, and F. Van Oystaeyen, "Pseudosymmetric braidings, twines and twisted algebras", J. Pure Appl. Algebra (2009). arXiv 0801.2055

[Staic 2004] M. D. Staic, "Pure-braided Hopf algebras and knot invariants", J. Knot Theory Ramifications 13:3 (2004), 385-400. MR 2005d:16069 Zbl 1106.16042 
[Suzuki 1982] M. Suzuki, Group theory, I, Grundlehren der Mathematischen Wissenschaften 247, Springer, Berlin, 1982. MR 82k:20001c Zbl 0472.20001

Received December 17, 2008. Revised June 26, 2009.

FLORIN PANAITE

Institute of MATHEMatics of The Romanian ACADEMY

P.O. BOX 1-764

014700 BUCHAREST

ROMANIA

Florin.Panaite@imar.ro

http://www.imar.ro/ fpanaite/

Mihai D. STAIC

Institute of MATHEMATICS OF THE Romanian ACADEMY

P.O. BOX 1-764

014700 BUCHAREST

ROMANIA

Current address:

DEPARTMENT OF MATHEMATICS

INDIANA UNIVERSITY

RAWLES HALL

BLOOMINGTON, IN 47405

UNITED STATES

mstaic@indiana.edu 